\title{
An Immunodominant Membrane Protein (Imp) of 'Candidatus Phytoplasma mali' Binds to Plant Actin
}

\author{
K. Boonrod, B. Munteanu, B. Jarausch, W. Jarausch, and G. Krczal \\ RLP AgroScience GmbH, AIPlanta-Institute for Plant Research, Breitenweg 71, 67435 Neustadt, Germany
}

Submitted 30 November 2011. Accepted 29 February 2012.

\begin{abstract}
The phytopathogenic, cell-wall-less phytoplasmas exhibit a dual life cycle: they multiply in the phloem of their host plant and in the body of their insect vector. Their membrane proteins are in direct contact with both hosts and are supposed to play a crucial role in the phytoplasma spread within the plant as well as by the insect vector. Three types of nonhomologous but highly abundant and immunodominant membrane proteins (IDP) have been identified within the phytoplasmas: Amp, IdpA, and Imp. Although recent results indicate that Amp is involved in vector specificity interacting with insect proteins such as actin, little is known about the interaction of IDP with the plant. We could demonstrate that transiently expressed Imp of ' $\mathrm{Can}$ didatus Phytoplasma mali' as well as the Imp without transmembrane domain $\left(\operatorname{Imp}{ }^{\Delta T m}\right)$ bind with plant actins in vivo. Moreover, in vitro co-sediment and binding assays showed that Escherichia coli-expressed recombinant $\operatorname{Imp}^{\mathbf{T m}}$-His binds to both G- and F-actins isolated from rabbit muscle. Transgenic plants expressing Imp- or $\mathrm{Imp}^{\Delta \mathrm{Tm}}$-green fluorescent protein did not exhibit any remarkable change of phenotype compared with the wildtype plant. These results indicate that Imp specifically binds to plant actin and a role of Imp-actin binding in phytoplasma motility is hypothesized.
\end{abstract}

Immunodominant membrane proteins (IDP) are very abundant among total cellular membrane proteins in most phytoplasmas. Genes encoding IDP were isolated from eight phytoplasmas (Kakizawa et al. 2004) which are enormously different in amino acid compositions and antigenicity. IDP are classified into three types: i) Imp, ii) IdpA (immunodominant membrane protein A), and iii) Amp (antigenic membrane protein) (Kakizawa et al. 2006; Morton et al. 2003). To date, only the possible biological function of Amp was studied. It was shown that Amp of onion yellows phytoplasma (OY) co-localized with the insect microfilaments of the visceral smooth muscle surrounding the intestinal tract (Suzuki et al. 2006). Moreover, the results of a pull-down assay revealed that Amp was co-precipitated with insect actin and myosin proteins. Interestingly, Amp-microfilament complexes were detected only in OY-transmitting leafhopper species. These results suggested that an interaction between the phytoplasma Amp and host proteins could be essential for phytoplasma transmission by insect vectors (Suzuki et al. 2006). Because phytoplasmas

Corresponding author: K. Boonrod; Telephone: (49)-6321-6711333; Fax: (49)-6321-6711313; E-mail: kajohn.boonrod@agroscience.rlp.de

* The $\boldsymbol{e}$-Xtra logo stands for "electronic extra" and indicates that one supplementary figure is published online and that Figures 1 and 6 appear in color online. can only survive in their insect vectors or in the phloem of their host plants, it would be interesting to investigate the biological function of IDP in sieve elements.

Imp is being considered to be a common ancestor of phytoplasma IDP (Kakizawa et al. 2009) because it was observed in the genomes of Western X (WX) (Liefting and Kirkpatrick 2003) and OY phytoplasma (Kakizawa et al. 2009) in addition to their predominant IDP genes. However, it is not known yet if Imp is expressed as a transmembrane protein in these phytoplasmas. In contrast, neither the ortholog of IdpA nor the ortholog of Amp was found in 'Candidatus Phytoplasma mali' (Kube et al. 2008). In addition to the difference in amino acid composition between Amp and Imp, it was shown that Amp was expressed and secreted out of the phytoplasma cells while Imp was trapped to the cell membrane (Barbara et al. 2002; Morton et al. 2003). According to previous studies, Amp and Imp are IDP; however, it is likely that Amp and Imp are different not only in gene composition but also at the protein level. In recently published results of preliminary experiments, it was shown that Imp without transmembrane domain of lime witches' broom phytoplasma interacted in vitro with actin and ATP synthase of leafhopper vectors proteins in vitro (Siampour et al. 2011). However, biological studies of Imp in plants are missing. In this report, we analyzed the actin-binding activity of Imp in planta and in vitro.

\section{RESULTS}

Imp co-localizes with actin cytoskeleton in planta.

To study the subcellular localization of Imp, a recombinant Imp with green fluorescent protein (GFP) fused to its $C$ terminus was transiently expressed under the control of a $35 \mathrm{~S}$ promoter in Nicotiana benthamiana using an Agrobacteriummediated expression system. The protoplasts were prepared from the infiltrated leaves and GFP fluorescence was visualized under a microscope using a GFP filter. The results showed that Imp-GFP was detected in the cytoplasm associated with a filamentous network (Fig. 1, upper panel). To ensure that this filamentous network was the actin cytoskeleton, we coexpressed Imp-GFP and the actin binding domain of the mouse Talin (mTalin) fused to red fluorescent protein (mTalinDsRed2) from the same vector, which would allow the two proteins to be expressed in the same cells and at the same time point. The mTalin is known to decorate the filamentous actin (Kost et al. 1998). The merge of Imp-GFP and mTailn-DsRed2 signal clearly indicated that Imp co-localized with the actin cytoskeleton. It must be noted that the co-expression of Imp without a transmembrane domain (Imp $\left.{ }^{\mathbf{T m}}\right)$-GFP and mTailnDsRed 2 gave the same result as shown by Imp-GFP (data not shown). To further confirm the actin-binding ability of Imp, we applied latrunculin B (LatB, an actin-disrupting drug) treatments to leaves co-infiltrated with Imp-GFP and mTalin- 
DsRed2. As a result, the protoplasts prepared from the LatBtreated leaves showed that the actin cytoskeleton was destroyed and that the expressed Imp-GFP as well as the mTalin-DsRed2 were dispersed in the cytoplasm (Fig. 1, lower panel). This result demonstrated that Imp did, indeed, bind actin filaments.

\section{Recombinant $\operatorname{Imp}^{\Delta \mathrm{Tm}}$ protein binds directly to $\mathbf{G}$-actin in vitro.}

The association of Imp with networks of actin filaments was further investigated in an attempt to elucidate the possible role of Imp in modulating the interactions of these cytoskeleton components. We preferred to use Imp ${ }^{\mathbf{\Lambda} m}$ for these studies because $\operatorname{Imp}^{\mathbf{\Lambda}}$ exhibited binding with actin similar to that of Imp (shown in transient expression assay). Furthermore, previous reports indicated that the Imp protein was trapped in the cell membrane and the C-terminal part behind the transmembrane domain that was exposed outside of the cell membrane. To obtain high amounts of protein, the $\operatorname{Imp}^{\mathbf{\Lambda} \mathrm{Tm}}$-His was expressed in Escherichia coli and purified (Fig. 2A and B). The actin binding ability of $\operatorname{Imp}^{\mathbf{\Lambda}}$-His was then assayed by an enzyme-linked immunosorbent assay (ELISA) and in co-sedimentation assays. The ELISA was performed to evaluate the interaction of $\operatorname{Imp}^{\mathbf{\Lambda} \mathrm{m}}$-His with G-actin. The result revealed that $\mathrm{Imp}^{\mathbf{\Lambda} \mathrm{Tm}}$-His binds to G-actin from rabbit muscle, which was coated on a microtiter plate in a concentration-dependent fashion (Fig. 2C). Moreover, bovine serum albumen (BSA), a negative control, did not bind to actin, thus indicating the specificity of $\mathrm{Imp}^{\mathbf{\Lambda} \mathrm{T}}$-His-actin binding.

\section{Co-sedimentation assay.}

The actin-binding of Imp was further investigated in vitro. In order to obtain further evidence for a direct interaction, a co-sedimentation assay was performed. The binding ability of $\mathrm{Imp}^{\mathbf{\Lambda} \mathrm{Tm}}$-His on F-actin was studied in low-speed co-sedimentation assays. The purified recombinant $\operatorname{Imp}^{\mathbf{\Lambda} \mathrm{Tm}}$-His was incubated for $1 \mathrm{~h}$ with polymerized F-actin, and the mixture was centrifuged at low speed. The pellets were then analyzed by sodium dodecyl sulfate polyacrylamide gel electrophoresis
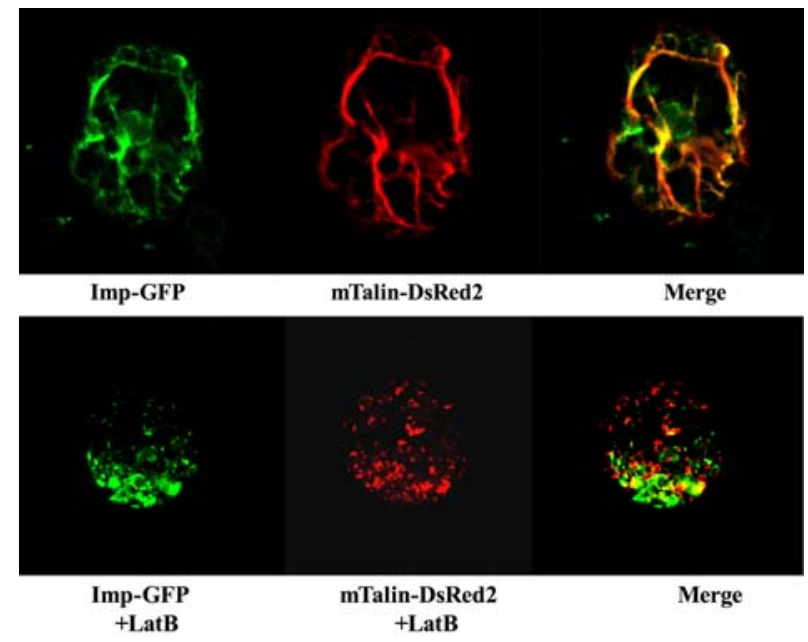

Fig. 1. Co-expression of Imp-green fluorescent protein (GFP) and mTalinDsRed2 fusion proteins in Nicotiana benthamiana and latrunculin B (LatB) treatment. Agrobacterium suspension carrying a double cassette of Imp-GFP and mTalin-DsRED2 was infiltrated into $N$. benthamiana plants. Two days post Agrobacterium spp.-mediated infiltration, the protoplasts were prepared from the infiltrated leaves and the subcellular localization of the fusion proteins was visualized by fluorescence microscopy. The colocalization of expressed Imp-GFP and mTalin-DsRED2 was judged by the presence of yellow filaments in the merge (upper panel). Imp-GFP and mTalin-DsRED2-labeled microfilaments are degraded in tissue treated for $24 \mathrm{~h}$ with $10 \mathrm{mM}$ LatB (lower panel).
(SDS-PAGE). The result in Figure 3 clearly showed that $\mathrm{Imp}^{\mathbf{\Lambda m}}$-His was found enriched in the pellet together with Factin, and no trace of aggregates of $\operatorname{Imp}^{\mathbf{\Lambda}}{ }^{\mathrm{Tm}}$-His was detected (data not shown), whereas the control BSA did not co-precipitate with F-actin. These data demonstrate that $\operatorname{Imp}^{\mathbf{\Lambda} \mathrm{Tm}_{-}}$-His interacts directly with $\mathrm{F}$-actin in vitro.

Our results showed that $\operatorname{Imp}^{\mathbf{\Lambda} \mathrm{Tm}}$-His binds G- and F-actin in vitro; therefore, we queried whether the binding of $\operatorname{Imp}^{\mathbf{\Lambda}} \mathrm{Tm}_{\text {-His }}$ to G-actin could inhibit the F-actin formation during polymerization. To elucidate this, $\operatorname{Imp}^{\mathbf{\Lambda}} \mathrm{Tm}_{\text {-His }}$ was preincubated with $\mathrm{G}$-actin prior to induction of polymerization. After centrifugation, the co-sediment pellets were analyzed in SDS-PAGE. The result indicated that the amount of F-actin was not decreased by increasing amounts of $\mathrm{Imp}^{\mathbf{\Lambda} \mathrm{Tm}}$-His, thus demonstrating that $\mathrm{Imp}^{\mathbf{\Lambda}} \mathrm{Tm}_{\text {-His }}$ did not inhibit the polymerization of actin (Fig. 4). Furthermore, the increasing amount of $\operatorname{Imp}^{\mathbf{\Lambda} m}$-His found in the co-sediment revealed that the binding of $\operatorname{Imp}^{\mathbf{\Lambda}}{ }^{\mathrm{Tm}}$-His to F-actin occurs in a dosage-dependent manner (Fig. 4).

\section{Stably expressed $\operatorname{Imp}$ and $\operatorname{Imp}^{\Delta \mathrm{Tm}}$ in transgenic plants do} not affect plant development.

Although the result of in vitro binding of Imp to actin clearly indicated that $\operatorname{Imp}^{\mathbf{\Lambda}}{ }^{\mathrm{Tm}}$-His did not inhibit F-actin polymerization, other possible effects related to this binding could not be

A

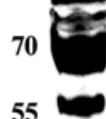

35

25
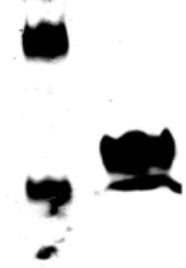

B

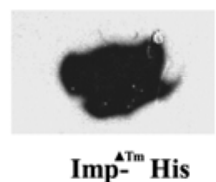

kDa M Imp- ${ }^{\text {Tim }}$ His

C

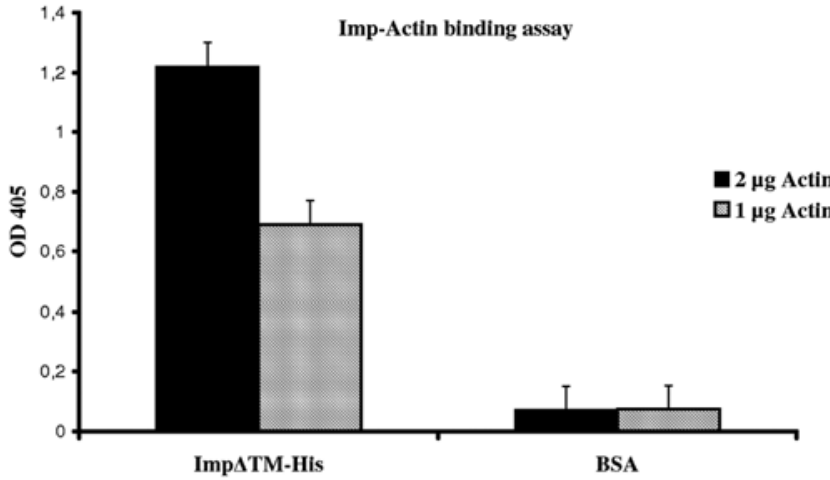

Fig. 2. Enzyme-linked immunosorbent assay experiment showing Escherichia coli-expressed $\operatorname{Imp}^{\mathbf{\Lambda}}{ }^{\mathrm{Tm}}$ bound to G-actin. $\operatorname{Imp}^{\mathbf{\Lambda}}{ }^{\mathrm{Tm}}$-His was expressed in E. coli (BL21 codon+). After purification, the purified protein was subjected to sodium dodecyl sulfate polyacrylamide gel electrophoresis. The purity and specificity of expressed $\mathrm{Imp}^{\mathbf{\Lambda}} \mathrm{Tm}_{\text {-His }}$ were determined by $\mathbf{A}$, Coomassie Brilliant blue $\mathrm{G}$ staining and $\mathbf{B}$, Western blot analysis using anti-His antibody. G-actin from rabbit muscle (1 and $2 \mu \mathrm{g})$ was coated on a microtiter plate. After blocking, $2 \mu \mathrm{g}$ of purified $\operatorname{Imp}^{\mathbf{T}{ }^{\mathbf{T}}}$-His was added. Binding of Imp ${ }^{\mathbf{\Lambda} m}$-His was measured using anti-His antibody (Qiagen) followed by anti-mouse-conjugated alkaline phosphatase (Sigma). After adding substrate, the signal was read with the absorbance at $405 \mathrm{~nm}$. C, Bovine serum albumin was used as negative control. 
excluded. In order to investigate the effect of expressed Imp on plant development, we produced transgenic plants expressing Imp- and Imp ${ }^{\mathbf{\Lambda}}$-fused GFP. Because the transformation of apple, the main host of ' $\mathrm{Ca}$. P. mali', has not yet sufficiently established, we used transgenic $N$. benthamiana as the model system because it has been demonstrated previously that Nicotiana spp. can be experimentally inoculated with ' $\mathrm{Ca}$. P. mali' (Kube et al. 2008). As a control, we produced ' $\mathrm{Ca}$. P. mali'infected $N$. benthamiana plants by grafting a shoot of phytoplasma-infected $N$. occidentalis that showed very strong symptoms (provided by B. Schneider, Julius-Kühn-Institut, Dossenheim, Germany) to wild-type $N$. benthamiana. The successfully grafted $N$. benthamiana plants were analyzed by polymerase chain reaction (PCR) for phytoplasma infection and the phytoplasma symptoms were monitored. The results showed that the PCR-positive grafted plants (data not shown) exhibited typical symptoms of phytoplasma infection, including shoot proliferation, deformation of leaves, necrosis, and seedlessness (Fig. 5A to C). This result indicated that $N$. benthamiana could be used as a model plant for studying the effect of phytoplasma proteins on plant development.

The transgenic $N$. benthamiana plants expressing Imp and $\operatorname{Imp}{ }^{\mathbf{\Lambda}}$-GFP were then established and the expression of ImpGFP and Imp ${ }^{\mathbf{\Lambda} m}$-GFP of T1 generation from different lines was analyzed by Western blot analysis. The result showed that the transgenic plant lines expressed Imp-GFP and $\operatorname{Imp}^{\mathbf{\Lambda}} \mathrm{Tm}_{-}$GFP (Fig. 6A and B). The protoplasts from the transgenic plants were isolated and the GFP fluorescence was visualized under the microscope using GFP filter. The result in Figure 6C showed networks of expressed Imp-GFP- and Imp ${ }^{\mathbf{\Lambda}} \mathrm{Tm}_{-}$GFP-labeled microfilaments while the expression of a single-chain variable fragment fused with GFP as a negative control was accumulated in the cytoplasm. The monitoring of these plants from germination till flowering was compared with ' $\mathrm{Ca}$. P. mali'-infected $\mathrm{N}$. benthamiana. The Imp-expressing plants did not exhibit any remarkable change in phenotype or symptoms typical for phytoplasma infection (Fig. 5D), thus indicating that binding of Imp to plant actin did not affect plant development.

\section{DISCUSSION}

Imp was proposed to be an ancestor of phytoplasma membrane proteins. However, the biological meaning of this pro-

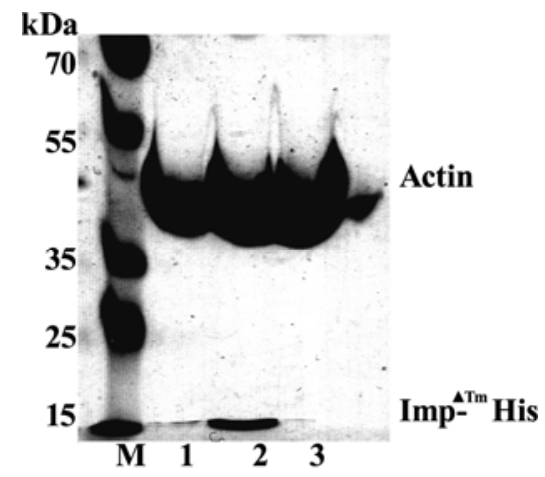

Fig. 3. $\operatorname{Imp}^{\mathbf{\Lambda m}}$ bound directly to F-actin in co-sedimentation assay. $\operatorname{Imp}^{\mathbf{\Lambda m}}$ $(1 \mu \mathrm{g})$ or a negative control (bovine serum albumin [BSA], $1 \mu \mathrm{g}$ ) were incubated with F-actin $(2 \mu \mathrm{g})$. Mixtures were centrifuged at 15,000 rpm (low-speed co-sedimentation) for $1 \mathrm{~h}$. Subsequently, pellets were analyzed by sodium dodecyl sulfate polyacrylamide gel electrophoresis followed by Coomassie Brilliant blue $\mathrm{G}$ staining. The presence of $\operatorname{Imp}^{\mathbf{\Lambda}} \mathrm{Tm}_{-H}$ is in the actin pellet after centrifugation indicated its binding to the actin while a negative control (BSA) did not co-sediment with F-actin. Lanes 1 is F-actin alone, lane 2 is the mixture of $\operatorname{Imp}^{\mathbf{A m}}$-His and F-actin, and lane 3 is the mixture of BSA and F-actin. tein was not known. Although Amp of OY phytoplasma was shown to bind to microfilaments of transmitting insects, its function in plants has not yet been described. Because phytoplasmas propagate in both insect and plant hosts, the study of the function of phytoplasma proteins expressed in plants is required. The result of transient expression of Imp-GFP and $\operatorname{Imp}^{\mathbf{A m}}$-GFP in $N$. benthamiana revealed that Imp and $\operatorname{Imp}^{\mathbf{T m}}$ localized along with the actin filament, which was confirmed by co-expression with actin-decorated protein, mTalinDsRed2. These results indicated that the actin-binding domain of Imp was located at the $\mathrm{C}$ terminus of the protein behind the transmembrane region. This finding supports the result of protein prediction that the C-terminal part of Imp is located outside of the phytoplasma membrane and, thus, is supposed to bind to host (plant) proteins.

The reaction of the plant to phytoplasma infection often involves growth deformations such as stunting, proliferation, or leaf distortion, in which cytoskeleton remodeling might be involved. Recently, it was shown that the transient expression of tobacco WLIM1 (an actin-binding protein [ABP] with an LIM domain related to animal Cys-rich proteins) in N. benthamiana plants induced a dramatic decrease of the overall number of actin filaments or bundles and a concomitant thickening of actin bundles (Thomas et al. 2006). However this phenomenon was not observed in $N$. benthamiana plants expressing Imp and Imp ${ }^{\mathbf{A m}}$ suggesting that the actin-binding of Imp did not induce actin cytoskeleton remodeling.

$\mathrm{ABP}$ are essential players in cytoskeleton dynamics by acting in cooperation to define the spatial separation and organization of filament branching, elongation, and nucleation, as well as actin assembly and disassembly processes (dos Remedios et al. 2003). This prompted us to further closely investigate the actin-binding function of Imp. The binding ability of $\operatorname{Imp}^{\mathbf{A T}}$ His to G-actin (result of ELISA) and F-actin (result of co-sediment assay) confirmed the result of the transient expression of Imp- and Imp ${ }^{\mathbf{A m}}$-GFP that Imp does, indeed, bind to actin filaments. Because binding of $\operatorname{Imp}^{\mathbf{\Lambda}}{ }^{\mathrm{Tm}}$-His to G-actin (in ELISA) did not inhibit the F-actin polymerization (in co-sedimentation assay), it is suggested that the actin-binding activity of Imp is a specific feature necessary for phytoplasma survival and is not involved in phytoplasma pathogenesis in plants. In plant cells,

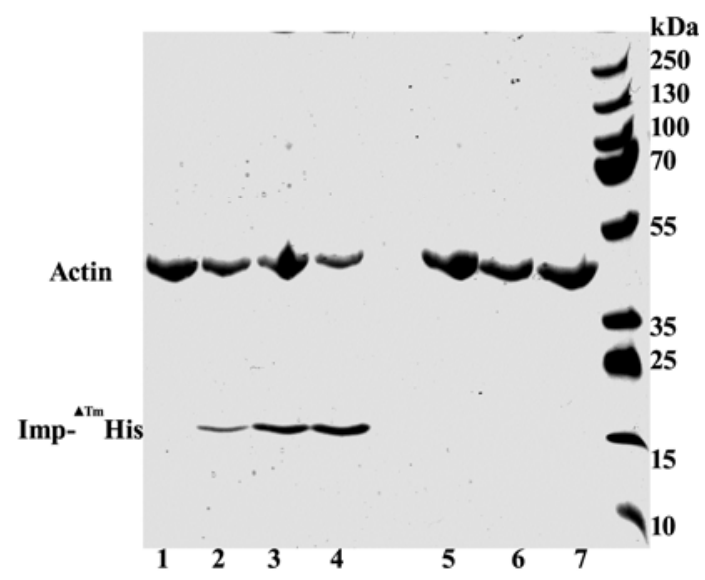

Fig. 4. $\mathrm{Imp}^{\mathbf{\Lambda} \mathrm{Tm}}$ did not inhibit $\mathrm{F}$-actin formation. Increasing concentrations of $\operatorname{Imp}^{\wedge \mathrm{Tm}}$-His $(0.5$ to $1 \mu \mathrm{g}$ ) or a negative control (bovine serum albumin [BSA], 0.5 to $1 \mu \mathrm{g})$ were incubated with G-actin $(0.5 \mu \mathrm{g})$ prior to induce actin polymerization. After inducing polymerization, the reactions were centrifuged at $15,000 \mathrm{rpm}$ and the pellets were analyzed by sodium dodecyl sulfate polyacrylamide gel electrophoresis followed by Coomassie Brilliant blue $\mathrm{G}$ straining. Lane 1 is polymerized actin as a negative control; lanes 2,3 , and 4 are $0.5,0.75$, and $1 \mu \mathrm{g}$, respectively, of $\operatorname{Imp}^{\mathbf{\Lambda}}{ }_{\text {-His; }}$ and lanes 5,6 , and 7 are $0.5,0,75$, and $1 \mu \mathrm{g}$, respectively, of BSA. 
the formation of higher-order actin structures is crucial to stabilize the organization of transvacuolar strands and maintain the overall cellular architecture (Shimmen et al. 1995). Therefore, inhibiting actin polymerization can change cellular morphology, inhibit cellular processes such as cell division, and even cause cells to undergo apoptosis (Haidle and Myers 2004). Moreover, cellular disruption of the equilibrium between actin monomers and actin filaments by overexpression of ABP or using actin cytoskeleton drugs (e.g., latrunculin or phalloidin) induces morphological and cytoarchitectural defects in plant cells (Gibbon et al. 1999; Staiger et al. 1994; Szymanski et al. 1999; Vidali et al. 2001). The result of transgenic plants expressing Imp- and $\mathrm{Imp}^{\mathbf{\Lambda}}{ }^{\mathrm{Tm}}$-GFP showed that expressed Imp- and Imp ${ }^{\boldsymbol{\Lambda} \mathrm{Tm}}$-GFP bound to actin filament but did not induce phytoplasma-like symptoms or defections of the plant phenotype. This result clearly indicated that the actinbinding activity of Imp neither interfered with actin polymerization nor disrupted the equilibrium between actin monomers and actin filaments. Moreover, possible detrimental effects of Imp on other plant proteins which could lead to phytoplasmalike symptoms could be excluded.

Although Amp was shown to bind to insect actin (Galetto et al. 2011; Suzuki et al. 2006), this binding was not reported to exhibit any negative effect on the life cycle of the host insect. Therefore, the results of our study support the hypothesis that binding of immunodominant protein to host actins could be a benefit for phytoplasma survival (probably for colonization, infection, and transmission) rather than the cause of its pathogenicity (Bertaccini and Duduk 2009).

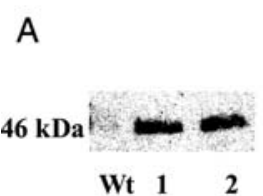

B

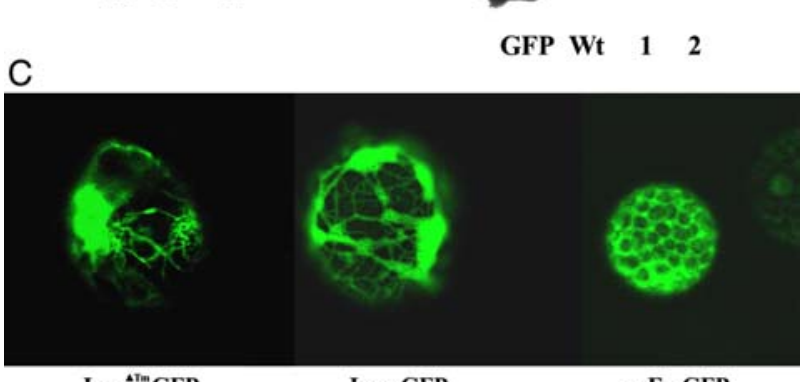

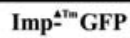

Imp-GFP

scFv-GFP

Fig. 6. Transgenic plants expressing Imp- and $\mathrm{Imp}^{\mathbf{\Delta} \mathrm{Tm}}$-green fluorescent protein (GFP). A, A leaf of transgenic plants expressing Imp- (A) and $\mathrm{Imp}^{\mathbf{\Lambda} \mathrm{Tm}}$-GFP (B) from two independent transgenic lines was extracted and subjected to sodium dodecyl sulfate polyacrylamide gel electrophoresis, followed by Western blotting. The presence of Imp- and Imp ${ }^{\mathbf{\Lambda} m_{-}}$-GFP was detected by using monoclonal anti-GFP-POD. Wt $=$ wild type Nicotiana benthamiana as a negative control. Lanes 1 and $2=$ transgenic $N$. benthamiana plant lines 1 and 2, respectively. B, Protoplasts were prepared from transgenic plants expressing Imp-and $\mathrm{Imp}^{\mathbf{\Lambda} \mathrm{T}}$-GFP and visualized under fluorescence microscopy. C, The protoplast prepared from a transgenic plant expressing a single-chain variable fragment $(\mathrm{scFv})$ fused with GFP (cytoplasmic expression) (K. Boonrod, unpublished data) was used as a negative control.
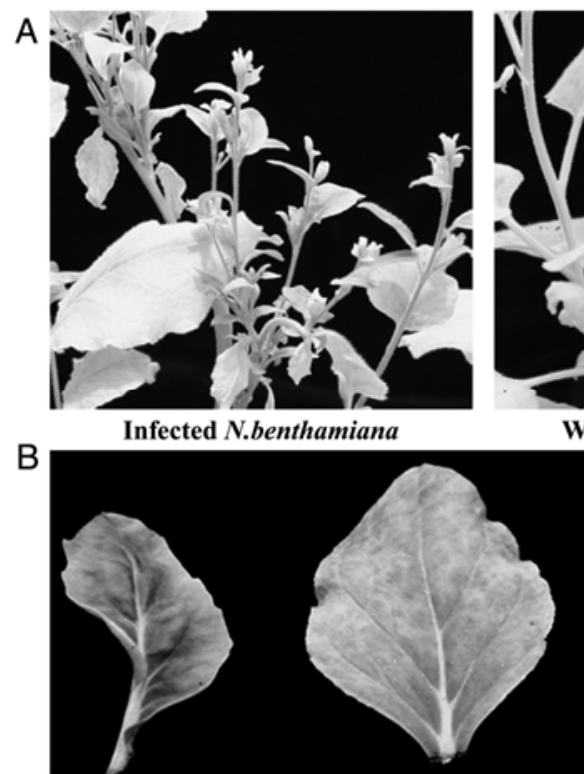

Infected $N$. benthamiana

C

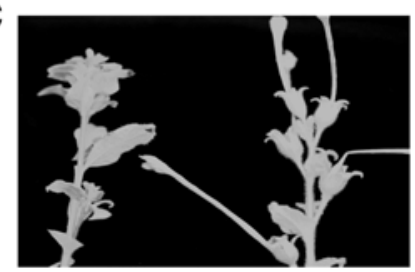

Infected $N$. benthamiana Wt N.benthamiana

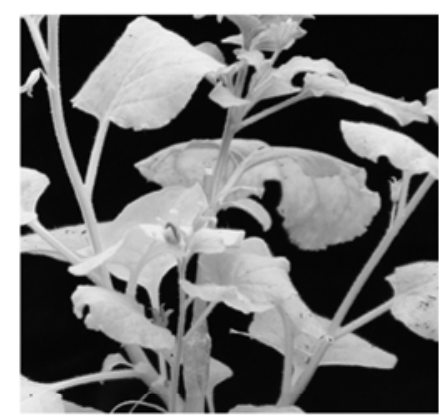

Wt. $N$. benthamiana

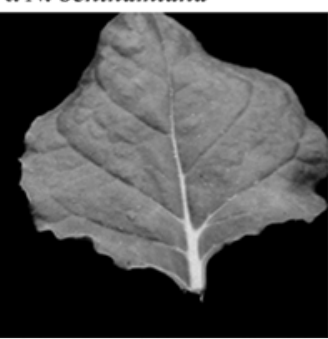

Wt $N$. benthamiana

D

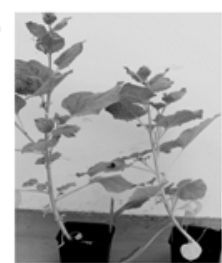

T1 Transgenic plant expressing Imp-GFP (left) and Imp $\Delta$ Tm-GFP (right)

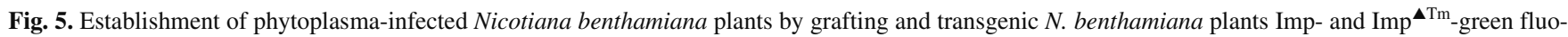
rescent protein (GFP). Shoots of phytoplasma-infected $N$. occidentalis were grafted on wild-type (Wt) healthy $N$. benthamiana. The successfully infected plants were analyzed by polymerase chain reaction as described. Phytoplasma-infected $N$. benthamiana plants exhibited phytoplasma typical symptoms: A, proliferation; B, chlorosis; and C, lack of seed. D, T1 generation of transgenic $N$. benthamiana plants expressing Imp- and Imp ${ }^{\mathbf{T m}}$-GFP. 
Some intracellular bacteria stimulate actin to drive the rocketing motility by its polymerization (Borisy and Svitkina 2000; Haglund and Welch 2011). In plants, inhibiting actin polymerization by treatment with either actin polymerization inhibitors or actin silencing (Liu et al. 2005) reduces both the movement protein (MP) virus particle trafficking of Tobacco mosaic virus (Sambade et al. 2008) and the efficiency by which MP accumulates in plasmodesmata (Kawakami et al. 2004; Wright et al. 2007). Proteomic analysis of phloem exudates of different plant species showed that levels of actin were found within the phloem sap of all species tested. Interestingly, the actin content in the phloem sap of Ricinus spp. hypocotyls was much higher than in a total tissue extract (Schobert et al. 1998). Moreover, a high amount of profilin, a potent regulator of actin polymerization, was found in the assimilate stream along with actin. It was suggested that this high profilin content prevented microfilament formation within the assimilate stream by producing monomeric G-actin. Therefore, plants may use the phloem system to deliver this key cytoskeleton protein to sink tissue (Schobert et al. 2000). Chaffey and Barlow (2002) reported that actin microfilaments were found alongside the cell plate and postulated that an actin-myosin contractile system is present at cell plates and sieve pores. We showed that Imp of ' $C a$. P. mali' binds to both G- and F-actin. Because phytoplasmas lack genes coding for movement, this feature could indicate a role of actin binding for the phytoplasma transport within the sieve elements and through the sieve plates. The binding of IDP to actin could support the movement of the phytoplasmas in the phloem and, thus, in the colonization of the plant host.

\section{MATERIALS AND METHODS}

\section{Nucleic acid extraction and PCR.}

Total nucleic acids from plant tissue (Malus and Nicotiana spp.) were extracted with a modified cetyltrimethylammonium bromide-based protocol as described by Jarausch and associates (2011). 'Ca. P. mali' was detected by PCR with standard procedures as referenced in Jarausch and associates (2011). The imp gene of ' $\mathrm{Ca}$. P. mali' PM19, a strain recently transmitted from field-collected Cacopsylla picta to healthy test plants of Malus $\times$ domestica (Jarausch et al. 2011), was amplified from the total DNA extract with primers $\mathrm{f} 318 \mathrm{~A} / \mathrm{r} 318 \mathrm{~B}$ as reported by Berg and associates (1999). PCR products were cloned into the pGEMT-easy vector according to the manufacturer's instructions (Promega, Madison, WI, U.S.A.), resulting in pGEMT-Imp.

\section{Plasmid constructs.}

$N c o$ I restriction sites were introduced at the $5^{\prime}$ and $3^{\prime}$ terminus of the Imp gene via PCR using primers 5'-GATAGCCAT GGAAGCAAATC- $3^{\prime}$ and $5^{\prime}$-CCCATGGGTTTCAAATC- ${ }^{\prime}$ and pGEM-T-Imp as a template. For Imp ${ }^{\mathbf{T m}}$, the first 96 nucleotides (code for first 32 amino acid residues) of Imp gene were deleted via PCR using primer 5'-GATAGCCATGGG TTCAA AAAG-3' (Supplementary Fig. 1). The purified PCR products were digested and cloned upstream fused to the GFP gene in pPZP200:GFP binary vector (Hajdukiewicz et al. 1994) for plant transformation, resulting in pPZP200:Imp-GFP and pPZP200:Imp ${ }^{\mathbf{T m}}$-GFP. In order to co-express Imp with an actin-binding mTalin protein (Kost et al. 1998), the whole expression cassette of a kanamycin-resistant gene (NPTII) at the left border of the pPZP200:Imp-GFP and pPZP200:Imp ${ }^{\mathbf{T m}}$ GFP plasmids was removed by digestion with $S m a \mathrm{I}$ and $K p n \mathrm{I}$ restriction enzymes. The expressed cassette of mTalin-DsRED2 (35S:Promoter-mTalin-DsRED2:Nos-terminator) was amplified from pCambia1390:mTalin-DsRed2 (kindly provided by E.
Blancaflor, The Samuel Roberts Noble Foundation) by a PCR, and $S m a \mathrm{I}$ and $\mathrm{KpnI}$ restriction sites were introduced at the $5^{\prime}$ and $3^{\prime}$ terminus. The PCR products were digested and then subcloned into the left border of pPZP200:Imp-GFP and pPZP200:Imp ${ }^{\mathbf{A m}}$-GFP, resulting in pPZP200:Imp-GFP/ mTalin-DsRED2 and pPZP200:Imp ${ }^{\mathbf{A m}}$-GFP/mTalin-DsRED2.

\section{Plant transformation.}

The plasmid was transformed into Agrobacterium tumefaciens ATHV by electroporation. The bacterial cell suspension was used for leaf disc transformation of $N$. benthamiana plants. Transgenic $N$. benthamiana plants were generated and maintained as described by Horsch and associates (1985).

\section{Transient protein expression in planta and protoplast preparation.}

Agroinfiltration was performed as previously described (Schöb et al. 1997). Briefly, a single colony of transformed agrobacteria was grown at $28^{\circ} \mathrm{C}$. Bacteria were centrifuged and the pellet was resuspended in induction media and grown overnight at $28^{\circ} \mathrm{C}$. The pellet of this overnight culture was resuspended in infiltration media up to an optical density at $600 \mathrm{~nm}\left(\mathrm{OD}_{600}\right)$ of 2.4 and was incubated at room temperature for at least $2 \mathrm{~h}$. The bacterial suspension was then infiltrated into leaves of each $N$. benthamiana plant using a 1-ml syringe. The infiltrated plants were incubated in the greenhouse for 2 days. The infiltrated leaves were collected 2 days after Agrobacterium-mediated infiltration. Two infiltrated leaves were cut in small strips (approximately $1 \mathrm{~mm}$ ) and placed in a $100-\mathrm{ml}$ flask containing $10 \mathrm{ml}$ of $1 \%$ cellulase (Onozuka R-10; Serva) and 0.2\% macerozyme (Onozuka R10; Serva, Heidelberg, Germany) in Murashige and Skoog medium (Murashige and Skoog 1962) supplemented with 0.4 $\mathrm{M}$ sucrose at $\mathrm{pH}$ 5.8. The mixture was incubated at room temperature for $2 \mathrm{~h}$ on a gyratory shaker $(20 \mathrm{rpm})$ to allow cell wall maceration. Intact protoplasts floating to the liquid surface were recovered with a Pasteur pipette and placed on a glass slide for fluorescence microscopy. Protoplasts were examined using a Zeiss Observer Z1 with LSM510 confocal laser-scanning head.

\section{LatB treatment.}

LatB (Adipogen, Hamburg, Germany) solution in dimethyl sulfoxide was freshly prepared and mixed with infiltration media to a final concentration of $10 \mathrm{mM}$. The mixture was syringe infiltrated into the abaxial leaf side.

\section{Cloning of $\operatorname{Imp}^{\Delta \mathrm{Tm}}$ gene in pET23a ${ }^{+}$.}

The Imp ${ }^{\mathbf{\Lambda m}}$ gene was amplified from pPZP:Imp ${ }^{\mathbf{\Lambda m}}$-GFP by introduction of a BamHI site at the $5^{\prime}$ terminus and an XhoI site at the $3^{\prime}$ terminus using primers $5^{\prime}$-GCGGATCCCCTTTT TCATCAAAAAGTAAACAAC- ${ }^{\prime}$ and $5^{\prime}$-GGTGCTCGAGTT TCAAATCTAAAACA-3'. The PCR products were directly cloned into a ptPCR vector (M. Wassenegger unpublished data), resulting in a ptPCR:BamHI-Imp ${ }^{\mathbf{\Lambda} \mathrm{m}}$-XhoI plasmid. In order to fuse $\operatorname{Imp}^{\mathbf{\Lambda m}}$ with hexahistidine (6XHis), they were subcloned into an expression vector, pET23a ${ }^{+}$(Novagen, Madison, WI, U.S.A.) at BamHI and XhoI sites, resulting in a pET23a $^{+}: \operatorname{Imp}^{\mathbf{A m}}$-His construct.

\section{Protein expression and purification.}

E. coli BL21 (DE3) codon plus (+) was transformed with $\mathrm{pET} 3 \mathrm{a}^{+}: \mathrm{Imp}^{\mathbf{A m}}$-His. Cells were grown in LB media supplemented with carbenicillin at $50 \mathrm{mg} /$ liter as antibiotics. At an $\mathrm{OD}_{600}=0.6$, protein expression was induced by adding isopropyl- $\beta$-D-thiogalactopyranoside to a final concentration of 1 $\mathrm{mM}$. After incubation at $37^{\circ} \mathrm{C}$ for $2 \mathrm{~h}$ or $14^{\circ} \mathrm{C}$ overnight, cells 
were harvested and resuspended in BugBuster Protein Extraction Reagent (Novagen; $10 \mathrm{ml} / \mathrm{g}$ of cells) containing $5 \mu \mathrm{l}$ of Benzonase (25 U/ $\mu \mathrm{l}), 10 \mathrm{mM}$ dithiothreitol (DTT), and one tablet of complete protease inhibitor (EDTA-free; Roche Applied Science, Indianapolis, IN, U.S.A.). The resuspended cells were incubated for $1 \mathrm{~h}$ at $4{ }^{\circ} \mathrm{C}$ under agitation. The lysate was centrifuged at $9.000 \times g$ for $10 \mathrm{~min}$ and the soluble fraction was purified by Ni-NTA agarose according to the manufacturer's protocol (Qiagen, Basel, Switzerland). The purified proteins were analyzed by SDS-PAGE followed by Coomassie Brilliant blue G (Sigma-Aldrich, St. Louis) staining or Western blot analysis.

\section{In vitro actin binding assay by ELISA.}

Actin (rabbit skeletal muscle, $2 \mathrm{mg} / \mathrm{ml}$; cytoskeleton) was prepared in low-salt buffer ( $5 \mathrm{mM}$ Tris- $\mathrm{HCl}$ [pH 8.0], $0.2 \mathrm{mM}$ $\mathrm{CaCl}_{2}, 0.2 \mathrm{mM} \mathrm{ATP}$, and $0.5 \mathrm{~mm} \mathrm{DTT}$ ) and 1 or $2 \mu \mathrm{g}$ of the actin solution was coated on a microtiter plate at $4^{\circ} \mathrm{C}$ overnight. Unspecific binding was blocked with 3\% BSA in phosphate-buffered saline buffer for $1 \mathrm{~h}$. Purified Imp ${ }^{\mathbf{T} m}$-His $(1 \mu \mathrm{g})$ was added and further incubated at room temperature for $1 \mathrm{~h}$. The plate was then washed and $100 \mu \mathrm{l}$ of primary anti-His antibodies (Qiagen) and anti-mouse conjugated with alkaline phosphatase (Sigma-Aldrich) were added. The protein binding was detected by adding freshly prepared substrate, p-nitrophenyl phosphate $(1 \mathrm{mg} / \mathrm{ml})$, and measuring the developed signal in a plate reader (Multiscan Ascent) at $405 \mathrm{~nm}$.

\section{Co-sedimentation assays.}

Rabbit muscle (cytoskeleton) was diluted at $0.05 \mu \mathrm{g} / \mu \mathrm{l}$ in a general actin buffer $(5 \mathrm{mM}$ Tris- $\mathrm{HCl}[\mathrm{pH} 8.0]$ and $0.2 \mathrm{mM}$ $\mathrm{CaCl}_{2}$ ). Polymerization was induced for $1 \mathrm{~h}$ at room temperature by the addition of actin polymerization inducer $(50 \mathrm{mM}$ $\mathrm{KCl}, 2 \mathrm{mM} \mathrm{MgCl}_{2}$, and $1 \mathrm{mM}$ ATP). Purified recombinant $\mathrm{Imp}^{\mathbf{\Lambda} \mathrm{T}}$-His protein or BSA was added to preassembled $\mathrm{F}$ Actin and the mixture was incubated at $4{ }^{\circ} \mathrm{C}$ for $1 \mathrm{~h}$. Proteins were pelleted at $15.000 \mathrm{rpm}$ for $1 \mathrm{~h}$. The pellets were analyzed by a SDS-PAGE followed by Coomassie Brilliant blue G (Sigma-Aldrich) staining.

To determine the inhibiting effect of Imp to polymerization of actin, increasing amounts of Imp were added to the polymerization reaction of actin. The reaction mixture was incubated at room temperature for $1 \mathrm{~h}$ and centrifuged at 15.000 rpm for $1 \mathrm{~h}$ at $4^{\circ} \mathrm{C}$. The pellets were analyzed by SDS-PAGE followed by Coomassie Brilliant blue staining.

\section{ACKNOWLEDGMENTS}

We thank M. Herdemertens and M. Braun for technical assistance, and B. Schneider for help in producing phytoplasma-infected N. benthamiana plants. This work was supported by a grant from the Stiftung RheinlandPfalz für Innovation.

\section{LITERATURE CITED}

Barbara, D. J., Morton, A., Clark M. F., and Davies D. L. 2002. Immunodominant membrane proteins from two phytoplasmas in the aster yellows clade (chlorante aster yellows and clover phyllody) are highly divergent in the major hydrophilic region. Microbiology 148:157-167.

Berg, M., Davis, D. L., Clark, M. F., Vetten, H. J., Maier, G., Marcone, C., and Seemüller, E. 1999. Isolation of the gene encoding an immunodominant membrane protein of the apple proliferation phytoplasma, and expression and characterization of the gene product. Microbiology 145:1937-1943.

Bertaccini, A., and Duduk, B. 2009. Phytoplasma and phytoplasma diseases: A review of recent research. Phytopathol. Mediterr. 48:355-378.

Borisy, G. G., and Svitkina, T. M. 2000. Actin machinery: Pushing the envelope. Curr. Opin. Cell Biol. 12:104-112.

Chaffey, N., and Barlow, P. 2002. Myosin, microtubules, and microfilaments: Co-operation between cytoskeletal components during cambial cell division and secondary vascular differentiation in trees. Planta 214:526-536

Dos Remedios, C. G., Chhabra, D., Kekic, M., Dedova, I. V., Tsubakihara, M., Berry, D. A., and Nosworthy, N. J. 2003. Actin binding proteins: Regulation of cytoskeletal microfilaments. Physiol. Rev. 83:433-473.

Galetto. L., Bosco, D., Balestrini, R., Genre, A., Fletcher, J., and Marzachi, C. 2011. The major antigenic membrane protein of 'Candidatus Phytoplasma asteris' selectively interacts with ATP synthase and actin of leafhopper vectors. PlosOne 6:e22571. doi:10.1371/journal.pone.0022571. Published online.

Gibbon, B. C., Kovar, D. R., and Staiger, C. J. 1999. Latrunculin B has different effects on pollen germination and tube growth. Plant Cell 11:2349-2363.

Haglund, C. M., and Welch, M. D. 2011. Pathogens and polymers: Microbe-host interactions illuminate the cytoskeleton. J. Cell Biol. 195:717.

Haidle, A., and Myers, A. 2004. An enantioselective, modular, and general route to the cytochalasins: Synthesis of L-696,474 and cytochalasin B. Proc. Natl. Acad. Sci. U.S.A. 101:12048-12053.

Hajdukiewicz, P., Svab, Z., and Maliga, P. 1994. The small, versatile PPZP family of Agrobacterium binary vectors for plant transformation. Plant Mol. Biol. 25:99-994.

Horsch, R. B., Rogers, S. G., and Fraley, R. T. 1985. Transgenic plants. Cold Spring Harb. Symp. Quant. Biol. 50:433-437.

Jarausch, B., Schwind, N., Fuchs, A., and Jarausch, W. 2011. Characteristics of the spread of apple proliferation by its vector Cacopsylla picta. Phytopathology. 101:1471-1480.

Kakizawa, S., Oshima, K., Nishigawa, H., Arashida, R., Miyata, S. I., Ugaki, M., Kishino, H., and Namba, S. 2004. Secretion of immunodominant membrane protein from onion yellows phytoplasma through the Sec protein-translocation system in Escherichia coli. Microbiology 150:135-142.

Kakizawa, S., Oshima, K., and Namba, S. 2006. Diversity and functional importance of phytoplasma membrane proteins. Trends Microbiol. $14: 254-256$.

Kakizawa, S., Oshima, K., Ishii, Y., Hoshi, A., Maejima, K., Jung, H.Y, Yamaji, Y., and Namba, S. 2009. Cloning of immunodominant membrane protein genes of phytoplasmas and their in planta expression. FEMS (Fed. Eur. Microbiol. Soc.) Microbiol. Lett. 293:92-101.

Kawakami, S., Watanabe, Y., and Beachy, R. N. 2004. Tobacco mosaic virus infection spreads cell to cell as intact replication complexes. Proc. Natl. Acad. Sci. USA. 101:6291-6296.

Kost, B., Spielhofer, P., and Chua, N. H. 1998. A GFP-mouse talin fusion protein labels plant actin filaments in vivo and visualizes the actin cytoskeleton in growing pollen tubes. Plant J. 16:393-401.

Kube, M., Schneider, B., Kuhl, H., Dandekar, T., Heitmann, K., Migdoll, A. M., Reinhardt, R., and Seemüller, E. 2008. The linear chromosome of the plant-pathogenic mycoplasma 'Candidatus Phytoplasma mali' BMC Genomics 9:306.

Liefting, L. W., and Kirkpatrick, B. C. 2003. Cosmid cloning and sample sequencing of the genome of the uncultivable mollicute, Western X-disease phytoplasma, using DNA purified by pulsed-field gel electrophoresis. FEMS (Fed. Eur. Microbiol. Soc.) Microbiol. Lett. 221:203-211.

Liu, J. Z., Blancaflor, E. B., and Nelson, R. S. 2005. The Tobacco mosaic virus 126-kilodalton protein, a constituent of the virus replication complex, alone or within the complex aligns with and traffics along microfilaments. Plant Physiol. 138:1877-1895.

Morton, A., Davies, D. L., Blomquist, C. L., and Barbara, D. J. 2003. Characterization of homologues of the apple proliferation immunodominant membrane protein gene from three related phytoplasmas. Mol. Plant Pathol. 4:109-114.

Murashige, T., and F. Skoog. 1962. A revised medium for rapid growth and bioassays with tobacco cultures. Physiol. Plant 15:473-497.

Sambade, A., Brandner, K., Hofmann, C., Seemanpillai, M., Mutterer, J., and Heinlein, M. 2008. Transport of TMV movement protein particles associated with the targeting of RNA to plasmodesmata. Traffic 9:20732088.

Schöb, H., Kunz, C., and Meins, F., Jr. 1997. Silencing of transgenes introduced into leaves by agroinfiltration: A simple, rapid method for investigating sequence requirements for gene silencing. Mol. Gen. Genet. 256:581-585.

Schobert, C., Baker, L., Szederkenyi, J., Grossmann, P., Komor, E., Hayashi, H., Chino, M., and Lucas, W. J. 1998. Identification of immunologically related proteins in sieve-tube exudate collected from monocotyledonous and dicotyledonous plants. Planta 206:245-252.

Schobert, C., Gottschalk, M., Kovar, D. R., Staiger, C. J., Yoo, B. C., and Lucas, W. J. 2000. Characterization of Ricinus communis phloem profilin, RcPRO1. Plant Mol. Biol. 42:719-730.

Shimmen, T., Hamatani, M., Saito, S., Yokota, E., Mimura, T., Fusetani, N., and Karaki, H. 1995. Roles of actin filaments in cytoplasmic 
streaming and organization of transvacuolar strands in root hair cells of Hydrocharis. Protoplasma 185:188-193.

Siampour, M., Galetto, L., Bosco, D., Izadpanah, K., and Marzachi, C. 2011. In vitro interaction between immunodominant membrane protein of lime witches' broom phytoplasma and leafhopper vector proteins. Bull. Insect. Suppl. 64:S149-S150.

Staiger, C. J., Yuan, M., Valenta, R., Shaw, P. J., Warn, R. M., and Lloyd, C. W. 1994. Microinjected profilin affects cytoplasmic streaming in plant cells by rapidly depolymerising actin microfilaments. Curr. Biol. 4:215-219.

Suzuki, S., Oshima, K., Kakizawa, S., Arashida, R., Jung, H. Y., Yamaji, Y., Nishigawa, H., Ugaki, M., and Namba, S. 2006. Interaction between the membrane protein of a pathogen and insect microfilament complex determines insect-vector specificity. Proc. Natl. Acad. Sci. U.S.A 103:4252-4257.

Szymanski, P. T., and Goyal, R. K. 1999. Calponin binds to the 20-kilodalton regulatory light chain of myosin. Biochemistry 38:3778-3784.

Thomas, C., Hoffmann, C., Dieterle, M., Van Troys, M., Ampe, C., and Steinmetz, A. 2006. Tobacco WLIM1 is a novel F-actin binding protein involved in actin cytoskeleton remodelling. Plant Cell 18:194-206.

Vidali, L., McKenna, S. T., and Hepler, P. K. 2001. Actin polymerization is essential for pollen tube growth. Mol. Biol. Cell. 12:2534-2545.

Wright, K. M., Wood, N. T., Roberts, A. G., Chapman, S., Boevink, P., Mackenzie, K. M., and Oparka, K. J. 2007. Targeting of TMV movement protein to plasmodesmata requires the actin/ER network: Evidence from FRAP. Traffic 8:21-31. 